\title{
Clinical Outcome of COVID 19 Patients After Hospitalization: Observational Study from a Tertiary Care Hospital, PAKISTAN
}

\section{Sadia Ishaque ( $\nabla$ sadiamir70@gmail.com )}

LIAQUAT NATIONAL HOSPITAL

\section{Beenish Syed}

Sindh institute of infectious diseases hospital and research center

\section{Saima Akhter}

Liaquat National Hospital and Medical College

\section{Talha Safeer}

Liaquat National Hospital and Medical College

\section{Atif Hashmi}

Liaquat National Hospital and Medical College

\section{Yousra Mansoor}

Liaquat National Hospital and Medical College

\section{Shahroz khawaja}

Liaquat National Hospital and Medical College

Noureen Durrani

Liaquat National Hospital and Medical College

\section{Research article}

Keywords: COVID-19, follow-up, discharged patients, outcomes

Posted Date: December 10th, 2020

DOI: https://doi.org/10.21203/rs.3.rs-122949/v1

License: (1) This work is licensed under a Creative Commons Attribution 4.0 International License. Read Full License 


\section{Abstract}

Introduction: The outbreak of novel coronavirus (COVID-19) is a public health emergency that had caused disastrous results in more than 100 countries.

Method: This observational study is conducted with aim to track the course of clinical outcomes of the course of COVID 19 patients after discharge. Demographics, baseline characteristics of COVID-19 patients discharged from May till August 2020, in Liaquat National hospital Karachi, Pakistan were collected and analyzed by reviewing the medical records retrospectively. Post-hospitalization data related to clinical outcomes were obtained by following up the patients for 4 weeks.

Results: Total 96 records of discharged patients were reviewed. Mean age of them was $53.83 \pm 12.89$ years with male predominance (78.1\%). Out of 96 patients, $19(19.8 \%)$ patients were asymptomatic at the time of discharge whereas $77(80.2 \%)$ had improved symptoms. The frequent symptoms at time of discharged was fatigue (69.8\%) followed by shortness of breath (34.4\%). Over the follow-up of 4 weeks, there was significant reduction in symptoms from discharge to 4th week excluding first week only. Patients were found to have significantly improved chest $x$-ray findings from discharge to first week of follow-up. Out of 77 symptomatic patients, 50(64.9\%) patients were found to be asymptomatic while $27(35.1 \%)$ were persistently symptomatic on fourth week of the discharge with most frequent symptom of fatigue $(33 \%)$ followed by shortness of breath (18\%).

Conclusion: There was significant recovery in most of the COVID 19 survivors after discharge, confirmed by reduction in the symptoms and radiological improvement.

\section{Introduction}

The outbreak of novel Corona virus (COVID-19) is a public health emergency that caused disastrous outcomes in more than 100 countries(1). The first confirmed case of COVID 19 in Pakistan was reported on 26th February 2020. As of 23rd October 2020, there have been about 326,216 confirmed cases with 309,646 recoveries and 6,715 deaths in the country (2). Karachi, the economical hub of Pakistan has maximum number of reported cases due to its dense population (3). Since then the number of cases, linear trend and deaths are increased, which is an alarming situation for a developing country, like Pakistan.

Globally, numerous studies have been conducted about this pandemic, many of them observed the course of disease during hospitalization (4-6). However, the number of recovered and discharged COVID19 patients kept increasing worldwide and the definite clinical outcomes of the patients with COVID-19 after discharge were scarcely described in the literature $(4,7)$.

To author's best knowledge, no data from Pakistan has been published or available to describe the clinical outcome of COVID 19 patients after discharge from hospital. This study will help present the data regarding clinical symptoms, and outcomes of COVID 19 patients after discharge which in turn will not 
only help to unravel the long term complications of COVID 19 but also help in the better management of COVID 19 patients.

The aim of this study was to track the course of clinical outcomes of COVID 19 patients after discharge from one of the biggest and busiest tertiary care hospital in Karachi, Pakistan.

\section{Methodology}

This prospective cohort study was done at Liaquat National Hospital, a tertiary care almost 700 bed hospital. Data from May 2020 till August 2020 was collected. Patients above the age of 18 years with confirmed COVID PCR were selected, patients were discharged from the hospital if afebrile for at least 3 days in the hospital or significant improvement of respiratory symptoms and radiological abnormalities on chest radiograph were included in the study. Those who either couldn't be contacted (i.e. lost to follow up after 2 weeks even after reminder calls) after discharge or refused to participate in the study were excluded from the study. The duration of data collection was from May 1st 2020 to August 31st 2020. The approval of the study was taken from institutional review board (IRB) and ethical review committee (ERC) of Liaquat National Hospital.

In-hospital data collection on the proforma was done at the time of discharge, in which demographic data, symptoms and complete blood count, inflammatory makers and chest $x$ ray at the time of discharge were noted. Post hospitalization data was obtained by weekly follow up for 2 weeks, and then week 3 and 4 data was obtained via phone calls and recorded on the proforma.

All the data of continuous variables were tested for normality using Shapiro-Wilk test. Based on normality, mean or median ranks were calculated. Categorical data were presented as percentages.

\section{Data Analysis:}

The collected data were entered in Statistical Package for Social Sciences (Version 26.0, IBM Inc., Armonk, USA) for statistical analysis. Categorical variables were expressed in frequency and percentages. Continuous variables were presented as mean \pm standard deviation or median with range as appropriate. Normality of the data was assessed using Shapiro-Wilk test with p-value $>0.05$ was considered as normally distributed. Decrease in proportion of patients with specific symptoms from discharge to fourth week of post-discharge was determined using Cochrane Q-test. Dunn's test was applied for post-hoc pairwise comparison. Participants' characteristics and laboratory investigations were compared among symptomatic and asymptomatic patients and those with persistent and resolved symptoms. MannWhitney $\mathrm{U}$ test for ratio variables and Chi-square test for categorical variables were used. Wilcoxon signtest was also applied to compare number of symptoms among symptomatic patients at the time of discharge and 4th week of discharge. McNemar's test was applied to assess difference in proportion of patients with improved $x$-ray on discharge day and a week after discharge. Two tailed p-value less than 0.05 was defined as statistically significant.

\section{Results}


Total 96 records of discharged patents were reviewed. The overall mean age of discharged patients was $53.83 \pm 12.89$ years. Median hospital stay was $8(I Q R=6-11)$ days. Majority of the discharged patients were males $(n=75,78.1 \%)$ and nearly quarter of the patients had no co morbid $(n=26,27.1 \%)$. The commonest co morbid condition among discharged patients was diabetes $(n=48,50 \%)$ followed by hypertension $(n=43,44.8 \%)$, ischemic heart disease $(n=16,16.7 \%)$, asthma $(n=7,7.3 \%)$, chronic kidney disease $(n=2,2.1 \%)$, chronic obstructive pulmonary disease $(n=2,2.1 \%)$, cerebrovascular accident $(n=2$, 2.1\%), chronic liver disease $(n=1,1 \%)$, depression $(n=1,1 \%)$, brain tumor $(n=1,1 \%)$, psoriasis $(n=1,1 \%)$, Parkinson's syndrome $(n=1,1 \%)$, hypothyroidism $(n=1,1 \%)$. Almost half of the discharged patients were initially admitted to ward $(n=49,51 \%)$, and remaining were initially admitted to high dependency unit ( $n$ $=30,31.3 \%)$ and intensive care unit $(n=17,17.7 \%)$. On last hospitalization day, median NLR, ALC, CRP levels, LDH levels, d.dimer, ferritin was $4($ IQR = 3-7), 1606 (IQR = 1030-2369), $1.26($ IQR = 0.53-3.04), $385(\mathrm{IQR}=322.5-504.75), 1.07(\mathrm{IQR}=0.66-2.68), 791(\mathrm{IQR}=496-1186)$ respectively.

25 (26\%) patients had normal chest $x$-ray findings on discharge day whereas remaining $(n=71,74 \%)$ patients had improved $x$-ray with infiltrates. One week after discharge, 30 patients showed normal $x$-ray which was statistically significant $(p=0.031)$. Out of 96 discharged patients, $77(80.2 \%)$ patients had improved symptoms at the time of discharge with most frequent symptom of fatigue $(n=67,69.8 \%)$ followed by shortness of breath $(n=33,34.4 \%)$, cough $(n=22,22.9 \%)$ and had two liters of oxygen requirement $(n=22,22.9 \%) .22(22.29 \%)$ patients who were discharged on two liters of oxygen requirement needed one liter of domiciliary oxygen. Patient who had no symptoms at the time of discharge had significantly lower median length of stay than patients who discharged with improve symptoms ( 6 days vs 9 days) $(p=0.001)$. Patients' age $(p=0.160)$, gender $(p=0.757)$, co-existence of any co morbidity $(p=0.622)$, specific co morbidities such as diabetes $(p=0.442)$, hypertension $(p=0.437)$, ischemic heart diseases $(n=0.182)$ were not significantly different among patients who were symptomatic and asymptomatic at the time of discharge. Patients who were either symptomatic or asymptomatic on discharge day also did not significantly differ on basis of laboratory findings on discharge day which included following laboratory investigations; CRP levels $(p=0.372)$, d.dimer $(p=$ $0.290), \operatorname{LDH}(p=0.850)$, ferritin $(p=0.825)$ NLR $(p=0.721)$ and ALC $(p=0.854)$.

Out of 19(19.8\%) patients who were asymptomatic at the time of discharge, none developed symptoms later either. Out of 77 patients who were symptomatic at the time of discharge, 27(35.1\%) were persistently symptomatic till fourth week whereas 50(64.9\%) of these patients became asymptomatic on fourth week. Patients' age $(p=0.072)$, gender $(p=0.413)$, co-existence of any comorbidity $(p=0.581)$, particularly comorbid conditions like diabetes $(p=0.345)$, hypertension $(p=0.510)$, ischemic heart diseases $(p=0.294)$ were not significantly different among patients who were persistently symptomatic and found to be asymptomatic on fourth week. Among symptomatic patients, the median number of symptoms per patients at discharge day was 2 (range: 1-4) whereas at fourth week median number of symptoms among these patients was 0 (range: $0-3$ ). The lower number of symptoms at fourth week compared to discharge time was statistically significant $(p<0.001)$. 
Figure 1 displays pattern of symptoms persistence from first week to fourth week post-discharge. The most commonly persisted symptom till fourth week was fatigue $(n=22,33 \%)$, followed by shortness of breath $(n=6,18 \%)$, cough $(n=1,5 \%)$ and 1 liter of oxygen requirement $(n=1,5 \%)$. Graphical pattern shows that there was decrease in symptoms including cough $(p<0.001)$, fatigue $(p<0.001)$, shortness of breath $(p<0.001)$ and 1 liters of oxygen requirement $(p<0.001)$ from discharge till fourth week which was also found to be statistically significant when tested with Cochrane Q-test. On post-hoc multiple comparison test, it was found that all of the symptoms significantly decreased from discharge day to every week excluding first week only.

\section{Discussion}

We systematically tracked the post hospitalization symptoms of the COVID 19 patients up till 4 weeks to see their pace of recovery.

Like other studies all patients were on a course of recovery $(4,7-9)$. Similar to Carfi et al our patient's age ranged from 52.9-58.1 years with male predominance $(7,9)$. The commonest co morbid in our cluster was diabetes; however unlike Balachander et al, we found no association of co-morbidities with presence of symptoms at the time of discharge (10). Most of the discharged patients were initially admitted to ward. The average length of stay in the hospital in our study was 8 days, while in a prospective cohort study done by Wang et al and Carfi et al showed longer hospital stay $(9,11)$. The most common symptoms at the time of discharge were fatigue and shortness of breath.

There were 77 patients who had improved symptoms at the time of discharge while 19 were completely asymptomatic. There was no statistical difference in the age, length of hospital stay, co morbidities, and laboratory finding among patients who were symptomatic and asymptomatic at the time of discharge. As observed in studies done by Luo et al and Wang et al in china and, in our study most of the patients from symptomatic group (64.9\%) became asymptomatic at the end of 4 th week $(4,7,8)$. while Garrigues et al showed persistent of post hospitalization symptoms for longer period of time (8). The most common symptoms that persisted at 4th week were fatigue and shortness of breath, this is inline with other studies which have also reported the commonest symptoms post hospitalization were fatigue and shortness of breath $(8,9)$.

Like other studies, there was a significant improvement in chest radiological findings in most the patients $(4,7)$.

At the end of the 4 th week most of the patients had returned to their normal life activity.

This observational study is so far the only study from Pakistan regarding the post hospitalization features of COVID 19.

Limitations of our study includes, small sample size from single center, follow up data up to 4 weeks only and limited data on quality of post COVID life. 


\section{Conclusion}

In our follow up study, we found the statistically significant recovery in most of the COVID 19 survivors after discharge, confirmed by reduction in the symptoms and radiological improvement. There is not statistically significant difference in the age, length of hospital stay, co morbidities, and laboratory findings among patients who were symptomatic and asymptomatic at the time of discharge.

Longer follow up with objective assessment of symptoms perseverance and quality of life scores is necessary to elucidate the physiological and psychological effects of COVID 19 on recovered patients. This will help us to design and improve rehabilitation system to boost COVID 19 survivor's morale and help them to return to normalcy.

\section{Declarations}

\section{Ethics approval and consent to participate}

The approval of the study was taken from institutional review board (IRB) and ethical review committee (ERC) of Liaquat National Hospital.

\section{Consent for publication}

Not applicable

\section{Availability of data and materials}

The datasets used and/or analyzed during the current study are available from the corresponding author on reasonable request

\section{Competing interests}

The Authors declare no Conflict of Interest.

\section{Funding}

None

\section{Author Contribution}

a. SA conceptualized the idea, data collection, data entry, manuscript writing.

b. BS developed the idea, data collection.

C. SA data cleaning.

d. AH data cleaning.

e. TS data collection.

f. YM data collection. 
g. S data collection.

h. ND data cleaning, data analysis.

\section{Acknowledgement}

We would like to acknowledge the contributions of Dr. Saleha Shahzad and Dr Sobia Ali for continue support and manuscript review. We would also like to acknowledge Kiran Akhtar for providing the support during data collection.

\section{References}

1. Sarwar S, Waheed R, Sarwar S, Khan A. COVID-19 challenges to Pakistan: Is GIS analysis useful to draw solutions? Science of The Total Environment. 2020;730:139089.

2. Government of Pakistan. Pakistan Statistics 2020 [Available from: https://covid.gov.pk/.

3. Noreen N, Dil S, Niazi S, Naveed I, Khan N, Khan F, et al. COVID 19 Pandemic \& Pakistan; Limitations and Gaps. 2020;1(4).

4. Liang W-H, Guan W-J, Li C-C, Li Y-M, Liang H-R, Zhao Y, et al. Clinical characteristics and outcomes of hospitalised patients with COVID-19 treated in Hubei (epicentre) and outside Hubei (non-epicentre): a nationwide analysis of China. Eur Respir J. 2020;55(6):2000562.

5. Swann OV, Holden KA, Turtle L, Pollock L, Fairfield CJ, Drake TM, et al. Clinical characteristics of children and young people admitted to hospital with covid-19 in United Kingdom: prospective multicentre observational cohort study. 2020;370.

6. COVID TC, Team R. Severe Outcomes Among Patients with Coronavirus Disease 2019 (COVID-19)United States, February 12-March 16, 2020. MMWR Morb Mortal Wkly Rep. 2020;69(12):343-6.

7. Luo S. A follow-up study of recovered patients with COVID-19 in Wuhan, China. International Journal of Infectious Diseases. 2020;99:2.

8. Garrigues E, Janvier P, Kherabi Y, Le Bot A, Hamon A, Gouze H, et al. Post-discharge persistent symptoms and health-related quality of life after hospitalization for COVID-19. Journal of Infection.

9. Carfi A, Bernabei R, Landi F. Group ftGAC-P-ACS. Persistent Symptoms in Patients After Acute COVID19. JAMA. 2020;324(6):603-5.

10. Balachandar V, Mahalaxmi I, Subramaniam M, Kaavya J, Senthil Kumar N, Laldinmawii G, et al. Follow-up studies in COVID-19 recovered patients - is it mandatory? Science of The Total Environment. 2020;729:139021.

11. Wang X, Xu H, Jiang H, Wang L, Lu C, Wei X, et al. Clinical features and outcomes of discharged coronavirus disease 2019 patients: a prospective cohort study. QJM: An International Journal of Medicine. 2020;113(9):657-65. 


\section{Tables}

Table 1

Comparison of patients' characteristics and laboratory investigations on discharge day between symptomatic and asymptomatic patients on discharge day

\begin{tabular}{|c|c|c|c|}
\hline Study Variables & $\begin{array}{l}\text { Symptomatic on discharge } \\
(n=77)\end{array}$ & $\begin{array}{l}\text { Asymptomatic on discharge } \\
(n=19)\end{array}$ & p-value \\
\hline Age (in years)\# & $54.75 \pm 12.03$ & $50.11 \pm 15.47$ & 0.160 \\
\hline 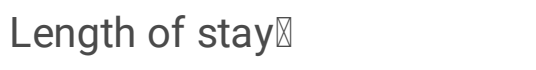 & $9(6-12)$ & $6(5-7)$ & 0.001 \\
\hline Male (\%) & $61(79.2)$ & $14(73.7)$ & $\bigotimes 0.757$ \\
\hline Any comorbidity (\%) & $57(74)$ & $13(68.4)$ & 0.622 \\
\hline Hypertension (\%) & $36(46.8)$ & $7(36.8)$ & 0.437 \\
\hline Diabetes (\%) & $40(51.9)$ & $8(42.1)$ & 0.442 \\
\hline Ischemic heart diseases (\%) & $15(19.5)$ & 1 & 0.182 \\
\hline CRPУ & $1.23(0.51-3.02)$ & $1.8(0.82-4)$ & 0.372 \\
\hline D. dimer $\bigotimes$ & $1.09(0.71-2.86)$ & $0.78(0.53-2.20)$ & 0.290 \\
\hline LDH囚 & $386(329-501)$ & $379(300-521)$ & 0.850 \\
\hline Ferritin $\bigotimes$ & $782(462-1203)$ & $881(536-1120)$ & 0.825 \\
\hline NLR】 & $4(2.5-7.0)$ & $5(3-7)$ & 0.721 \\
\hline ALCD & 1586 (1019-2461) & $1666(1235-2200)$ & 0.854 \\
\hline
\end{tabular}


Table 2

Patients' characteristics and laboratory investigations on discharge day among persistently symptomatic and asymptomatic patients on fourth week

\begin{tabular}{|c|c|c|c|}
\hline Study Variables & $\begin{array}{l}\text { Persistently symptomatic } \\
(\mathrm{n}=27)\end{array}$ & $\begin{array}{l}\text { Symptoms resolved } \\
(n=50)\end{array}$ & p-value \\
\hline Age (in years)/\# & $58.11 \pm 13.33$ & $52.94 \pm 10.98$ & 0.072 \\
\hline Male (\%) & $20(74.1)$ & $41(82)$ & 0.413 \\
\hline Any comorbidity (\%) & $21(77.8)$ & $36(72)$ & 0.581 \\
\hline Hypertension (\%) & $14(51.9)$ & $22(44)$ & 0.510 \\
\hline Diabetes (\%) & $16(59.3)$ & $24(48)$ & 0.345 \\
\hline Ischemic heart disease (\%) & 7 (25.9) & $8(16)$ & 0.294 \\
\hline CRPУ & $0.70(0.39-2.79)$ & $1.41(0.68-3.08)$ & 0.102 \\
\hline D. dimer囚 & $1.19(0.79-3.17)$ & $1.05(0.64-2.65)$ & 0.308 \\
\hline LDH囚 & $415(334-549)$ & $385.5(323.5-488.5)$ & 0.342 \\
\hline Ferritin $\rrbracket$ & $745(327-1209)$ & $791(498.75-1216.75)$ & 0.737 \\
\hline NLR囚 & $5(3-7)$ & $4(2-6)$ & 0.177 \\
\hline $\mathrm{ALCD}$ & $1430(786-2678)$ & 1599 (1076-2448) & 0.790 \\
\hline
\end{tabular}

\section{Figures}




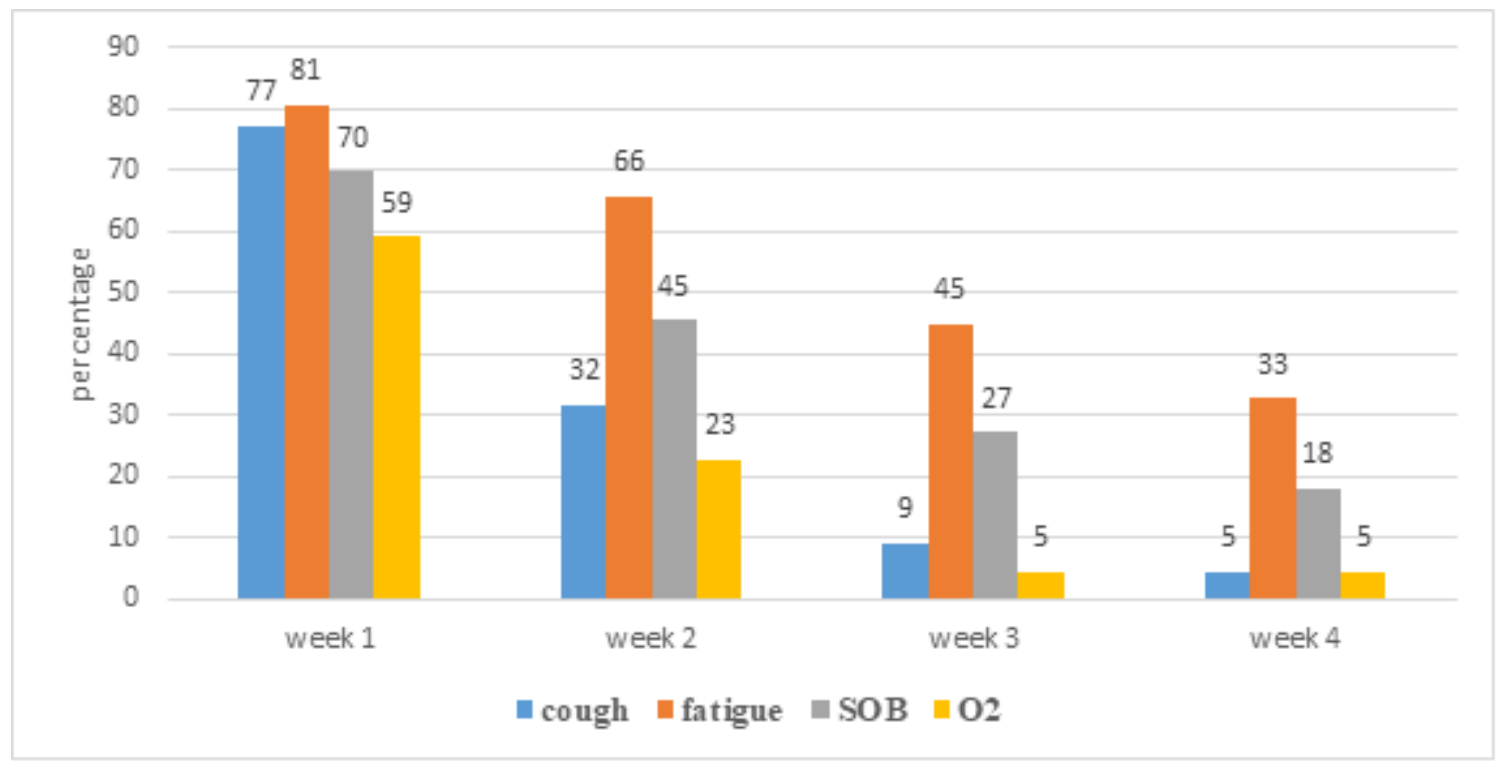

Figure 1

Pattern of specific symptom persistence in symptomatic patients

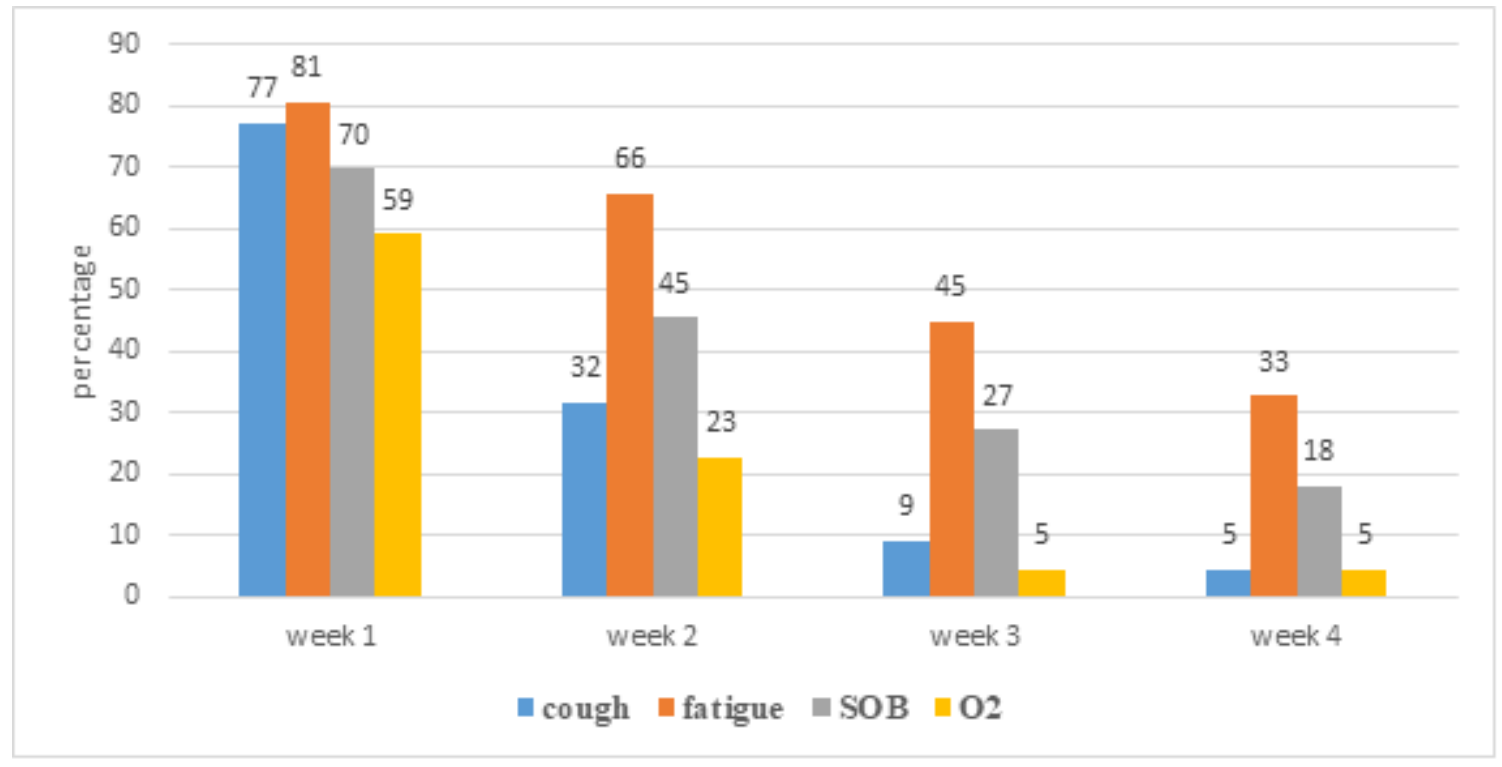

Figure 1

Pattern of specific symptom persistence in symptomatic patients 


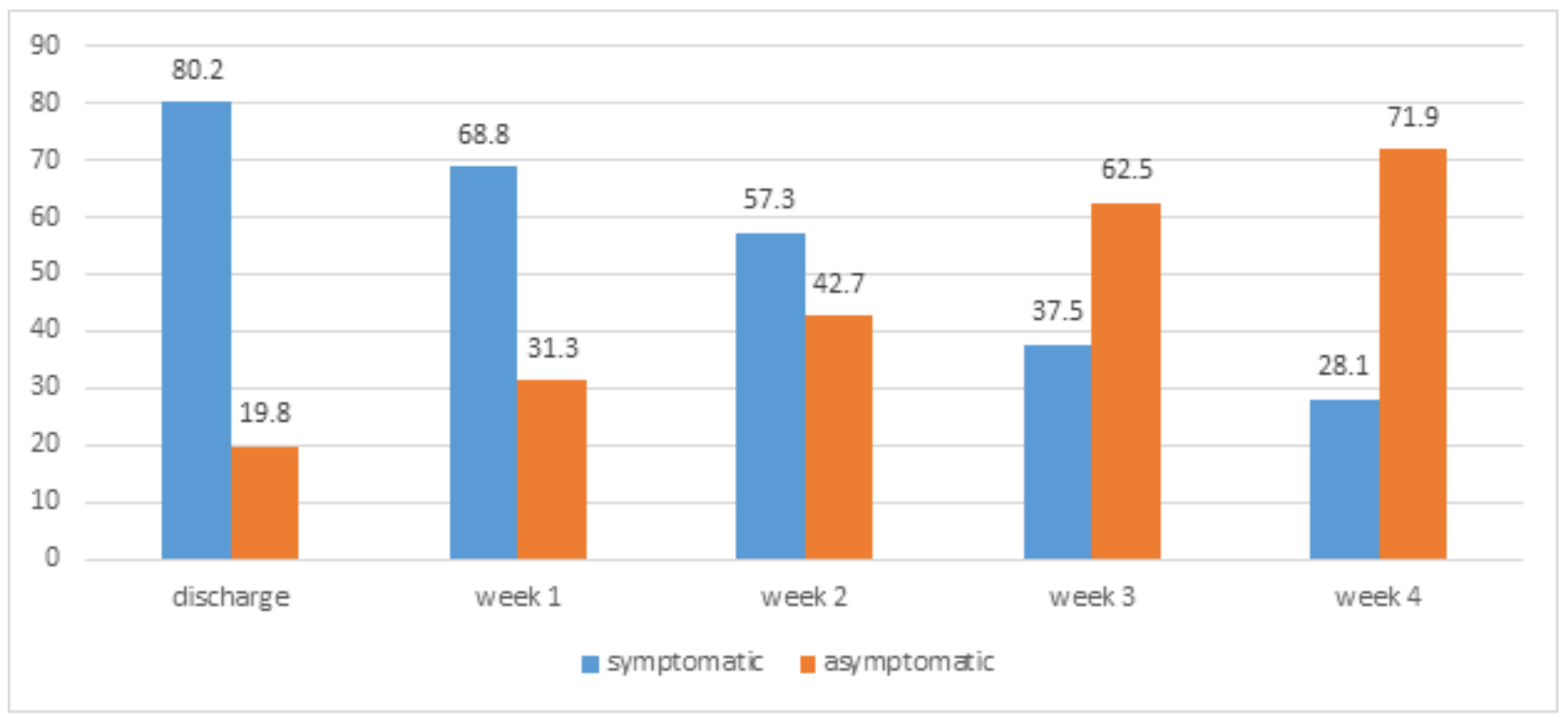

Figure 2

Pattern of symptoms resolution from discharge day to fourth week

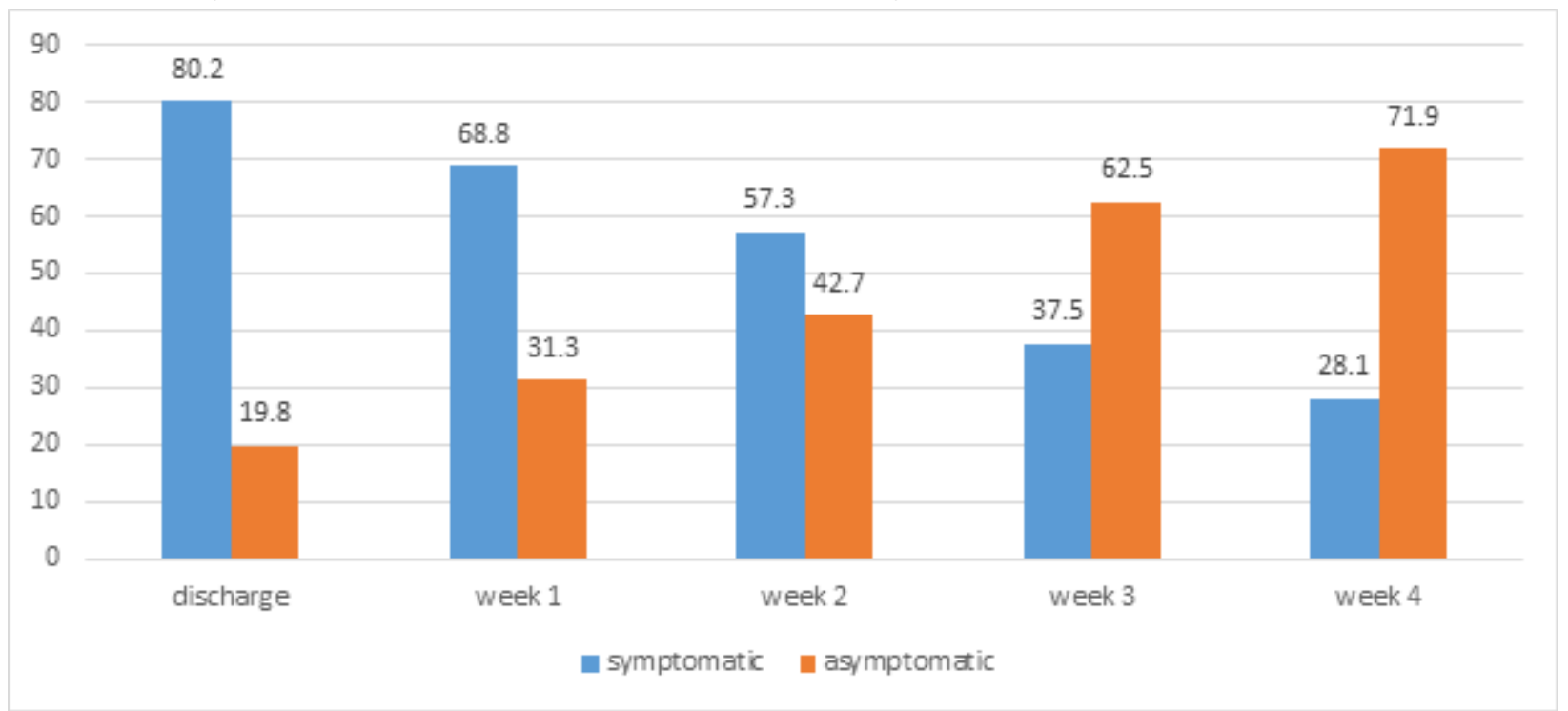

\section{Figure 2}

Pattern of symptoms resolution from discharge day to fourth week 